\title{
CODIMENSION TWO NONORIENTABLE SUBMANIFOLDS WITH NONNEGATIVE CURVATURE
}

\author{
YURIKO Y. BALDIN AND FRANCESCO MERCURI
}

(Communicated by David G. Ebin)

\begin{abstract}
We prove that a compact nonorientable $n$-dimensional submanifold of $\mathbf{R}^{n+2}$ with nonnegative curvature is a "generalized Klein bottle" if $n \geq 3$.
\end{abstract}

1. Introduction. In [1] we study isometric immersions of compact, orientable nonnegatively curved $n$-manifolds in $\mathbf{R}^{n+2}$. The aim of this paper is to study the nonorientable case. If $n=2$ there is an example of an isometric immersion of a flat Klein bottle in $\mathbf{R}^{4}$ (see [3]) and the results of [1] suggested that for $n \geq 3$ the "generalized Klein bottle" is, in fact, the only possible example. We will prove the following result.

THEOREM. Let $M^{n}, n \geq 3$, be a compact, nonorientable Riemannian manifold with nonnegative sectional curvatures and $f: M^{n} \rightarrow \mathbf{R}^{n+2}$ an isometric immersion. Then there exists a $(n-1)$-dimensional manifold $N^{n-1}$ homotopy equivalent to $S^{n-1}$, such that

(1) the orientable covering of $M$ is diffeomorphic to $S^{1} \times N^{n-1}$ and the metric is locally a product;

(2) $M$ is diffeomorphic to a nonorientable bundle over $S^{1}$ with fibre $N^{n-1}$ and the metric is locally a product;

(3) the covering projection sends the fibres $N^{n-1}$ of $S^{1} \times N^{n-1}$ isometrically onto the fibres of the bundle $M \rightarrow S^{1}$.

2. Known facts. We will state now some results to be used in the proof of the theorem. For their proofs and related references see [1].

$M$ will denote a $n$-dimensional Riemannian manifold, $n \geq 3$, compact, connected, with nonnegative sectional curvatures, which admits an isometric immersion $f: M^{n} \rightarrow \mathbf{R}^{n+2}$.

2.1. If $M$ is orientable over a field $F$ then $\sum_{i=1}^{n-1} b_{i}(M ; F) \leq 2$, where $b_{i}(M ; F)=$ $\operatorname{dim} H_{i}(M ; F)$ is the $i$ th Betti number of $M$ with coefficients in $F$.

2.2. If $M$ is orientable, not simply connected, then $\pi_{1}(M)$ is cyclic, and if $n \geq 4$, $\pi_{1}(M) \cong Z$.

2.3. If $M$ is orientable and $\pi_{1}(M) \cong Z$ then there exists a compact $(n-1)$ dimensional manifold $N$, homotopy-equivalent to a sphere such that $M$ is diffeomorphic to $S^{\mathbf{1}} \times N$ and the metric is locally a product. In fact the universal covering of $M$ is isometric to $\mathbf{R} \times N$.

Received by the editors July 13, 1987.

1980 Mathematics Subject Classification (1985 Revision). Primary 53C40; Secondary 53C42. 


\section{Proof of the theorem.}

3.1. $\pi_{1}(M) \cong Z$. Let $\theta: \bar{M} \rightarrow M$ be the orientation covering of $M$. By $2.2 \pi_{1}(\bar{M})$ is cyclic. If $\pi_{1}(\bar{M})$ is finite then $b_{1}(M ; \mathbf{R})=0$ and, by $2.2, n=3$. But $M$ is not orientable and therefore $b_{3}(M ; \mathbf{R})=0$ which leads to $0=\chi(M)=1+b_{2}(M ; \mathbf{R}) \geq 1$. So $\pi_{1}(\bar{M}) \cong Z$. Now, since $\theta$ is a double covering we have the exact sequence

$$
0 \rightarrow Z \cong \pi_{1}(\bar{M}) \stackrel{\theta_{\#}}{\longrightarrow} \pi_{1}(M) \rightarrow Z_{2} \rightarrow 0 .
$$

It is not difficult to see that the only groups that fit such an exact sequence are $Z$, $Z \times Z_{2}$ and the semi-direct product $Z \ltimes_{\phi} Z_{2}$ where $\phi: Z_{2} \rightarrow \operatorname{Aut}(Z) \cong Z_{2}$ is the identity. In the latter two cases we will have respectively

$$
H_{1}(M ; Z) \cong Z \oplus Z_{2} \text { and } H_{1}(M ; Z) \cong Z_{2} \oplus Z_{2}
$$

and in both cases $b_{1}\left(M ; Z_{2}\right)=2$. By duality $b_{n-1}\left(M ; Z_{2}\right)=2$ and therefore $\sum_{i=1}^{n-1} b_{i}\left(M ; Z_{2}\right) \geq 4$ which contradicts 2.1 . Therefore $\pi_{1}(M) \cong Z$ and $\theta_{\#}$ is multiplication by \pm 2 .

3.2. $M$ is a fibre bundle over $S^{1}$ with connected fibre. By $2.3 \bar{M}$ is diffeomorphic to $S^{1} \times N$ and the metric is locally a product. Let $\bar{X}$ be a unitary vector field tangent to the $S^{1}$ factor. Then $\bar{X}$ is parallel and the only one up to a constant multiple (in fact, $H^{\mathbf{1}}(\bar{M}, \mathbf{R}) \cong \mathbf{R}$ is generated by a 1 -form dual to a parallel field). Let $\tau: \bar{M} \rightarrow \bar{M}$ be the nontrivial covering transformation and define a vector field in $M$ by

$$
\left.X(p)=\frac{1}{2}\left\{(d \theta)_{x} \bar{X}(x)+(d \theta)_{\tau(x)} \bar{X}(\tau(x))\right\}, \quad p=\theta^{\prime} x\right),
$$

and then $X$ is a well-defined parallel field. We want to show that $X \neq 0$. In fact if $X \equiv 0$ then $(d \theta)(\bar{X})$ defines a line field whose integral curves are projections of the integral curves of $\bar{X}$. More precisely consider $\theta^{-1}(p)=\{x, \tau(x)\}$ and let $\gamma, \sigma$ be the integral curves of $\bar{X}$ through $x$ and $\tau(x)$ respectively. If $\gamma \neq \sigma, \theta(\gamma)$ and $\theta(\sigma)$ represent the same closed curve in $M$ with opposite orientation. Let $\alpha$ be a curve from $x$ to $\tau(x)$. The loop $\gamma * \alpha * \sigma * \alpha^{-1}$ represent twice the generator of $\pi_{1}(\bar{M})$ and therefore is nonzero. But $\theta\left(\gamma * \alpha * \sigma * \alpha^{-1}\right)$ is a commutator in $\pi_{1}(M)$, since, by the above, $\theta(\sigma)=\theta(\gamma)^{-1}$. This leads to a contradiction since $\pi_{1}(M)$ is abelian and $\theta_{\#}: \pi_{1}(\bar{M}) \rightarrow \pi_{1}(M)$ is $1-1$. If $\gamma=\sigma$ a similar argument leads to the same contradiction.

So $X$ is a nonzero parallel field. The distribution $X^{\perp}$ is integrable and its leaves are the image, by $\theta$, of the leaves of $\bar{X}^{\perp}$, so in particular they are compact. Now it is shown in [2], that for a complete Riemannian manifold $M$, there exist:

(a) A maximal subspace $U$ of the space of parallel fields such that the leaves of $U^{\perp}$ are closed in $M$ (Proposition III.5).

(b) A riemannian fibration of $M$ on a $m$-dimensional flat torus, $m=\operatorname{dim} U$, whose fibres are the integral leaves of $U^{\perp}$ (Proposition III.6).

The space of parallel fields, in our case, is of dimension $\leq 1$ (since $H_{1}(M ; \mathbf{R}) \cong$ $\mathbf{R})$, so it is spanned by $X$. So, with the above notation $U=\operatorname{span}\{X\}$ and the claim is proved.

3.3. The fibres of $M \rightarrow S^{1}$ are homotopy spheres. Let $F$ be the fibre of the above fibration. Since $F$ is connected and $\pi_{1}(M) \cong Z$ (by 3.1), we have the exact sequence

$$
\pi_{j+1}\left(S^{1}\right) \rightarrow \pi_{j}(F) \rightarrow \pi_{j}(M) \rightarrow \pi_{j}\left(S^{1}\right) \rightarrow \cdots \rightarrow \pi_{1}(F) \rightarrow Z \rightarrow Z \rightarrow 0 .
$$


Therefore $\pi_{1}(F)=0$ and $\pi_{j}(F) \cong \pi_{j}(M) \cong \pi_{j}(\bar{M}) \cong \pi_{j}(N)$, for $j \geq 2(\bar{M} \cong$ $\left.S^{1} \times N\right)$, which prove 3.3 and the theorem.

4. Final remarks. The compact, nonorientable surfaces which admit metrics of nonnegative curvature are the flat Klein bottle and the projective plane $\mathbf{R} P^{2}$. As we mentioned in the introduction the flat Klein bottle admits an isometric immersion in $\mathbf{R}^{\mathbf{4}}$. It would be interesting to know if $\mathbf{R} P^{\mathbf{2}}$ admits an immersion in $\mathbf{R}^{4}$ with nonnegative curvature. Also it would be of interest to construct examples of "generalized Klein bottle" in $\mathbf{R}^{n+2}$ with nonnegative curvature.

\section{REFERENCES}

1. Y. Y. Baldin and F. Mercuri, Isometric immersions in codimension two with nonnegative curvature, Math. Z. 173 (1980), 111-117.

2. J. P. Bourguignon and E. Mazet, Sur la structure des variétés riemanniennes qui admettent des champs de vecteurs paralleles, Compositio Math. 24 (1972), 105-117.

3. C. Tompkins, A flat Klein bottle isometrically imbedded in Euclidean 4-space, Bull. Amer. Math. Soc. 47 (1941), 508.

Departamento de Matemática, Universidade Federal de SÃo Carlos, 13560SÃo CARLOS, BRASIL

IMECC-UNICAMP, C.P. 6155, 13081-CAMPINAS, BRASIL 